\title{
A New Five-Parameter MOS Transistor Mismatch Model
}

\author{
Teresa Serrano-Gotarredona and Bernabé Linares-Barranco
}

\begin{abstract}
A new five-parameter MOS transistor mismatch model is introduced capable of predicting transistor mismatch with very high accuracy for ohmic and saturation regions, including short-channel transistors. The new model is based on splitting the contribution of the mobility degradation parameter mismatch $\Delta \theta$ into two components, and modulating them as the transistor transitions from ohmic to saturation regions. The model is tested for a wide range of transistor sizes (30), and shows excellent precision, never reported before for such a wide range of transistor sizes, including short-channel transistors.
\end{abstract}

Index Terms - Analog circuit modeling, analog circuit simulation, analog VLSI design, mismatch modeling, transistor mismatch, transistor modeling..

\section{INTRODUCTION}

C HARACTERIZATION and simulation of MOS transistor mismatch is crucial for precision analog design. Mismatch models include two terms: 1) a size dependent and 2) a distance dependent term [1]. The distance dependent term can be compensated through layout techniques (such as common centroids [2]), and is consequently less critical for precise analog design. In this letter, we will concentrate on the size dependent term. Usually, statistical characterization parameters are extracted independently for different regions of operation [1], [3]. However, when very small transistor lengths are used and a very wide range of transistor sizes are considered, the traditional mismatch models provide poor fit between measured and predicted values. Parameters extracted for ohmic region do not predict correctly measurements for saturation, and vice versa. Consequently, for good mismatch prediction, a set of mismatch parameters had to be extracted for ohmic region, and another set for saturation. In this letter, we report on an extended mismatch model whose statistical parameters are extracted simultaneously from curves measured in the ohmic and saturation regions, and a unique set of five mismatch parameters is obtained valid for both regions of operation. As a result, an extraordinary fit between measured and predicted mismatch is obtained, for both regions of operation, and for a very wide range of transistor sizes, including minimum channel length transistors.

Manuscript received May 20 1999. The review of this letter was arranged by Editor E. Sangiorgi.

The authors are with the National Microelectronics Center, 41012 Sevilla, Spain (e-mail: bernabe@imse.cnm.es; www.imse.cnm.es/ bernabe).

Publisher Item Identifier S 0741-3106(00)00436-5.

\section{The New Mismatch Model}

An acceptable strong inversion large-signal transistor model for mismatch is [1], [3]

$$
\begin{aligned}
I_{D S} & =\beta \frac{V_{G S}-V_{T}\left(V_{S B}\right)-\frac{1}{2} V_{D S_{\mathrm{cff}}}}{1+\theta\left(V_{G S}-V_{T}\left(V_{S B}\right)\right)} V_{D S_{\mathrm{cff}}} \\
V_{T}\left(V_{S B}\right) & =V_{T 0}+\gamma\left[\sqrt{\phi+V_{S B}}-\sqrt{\phi}\right]
\end{aligned}
$$

where

$$
\begin{array}{ll}
V_{D S_{\text {cff }}}=V_{D S} & \text { in ohmic region; } \\
V_{D S_{\text {cff }}}= & \text { in saturation; } \\
V_{G S}-V_{T}\left(V_{S B}\right) & \\
\beta=\mu C_{o x} W / L & \text { current gain factor; } \\
V_{T 0} & \text { zero-bias threshold voltage; } \\
\gamma & \text { bulk threshold parameter; } \\
\theta & \text { mobility degradation parameter. }
\end{array}
$$

However, for short-channel transistors, the effect of source and drain series resistance as well as carriers velocity saturation are important for mismatch, but are not modeled in (1). The effect of drain and source series resistances can be included in (1) by replacing $V_{G S}$ by $V_{G S}-I_{D S} R_{S}$ and $V_{D S}$ by $V_{D S}-I_{D S}\left(R_{S}+\right.$ $\left.R_{D}\right)$. After neglecting high-order terms, an equation similar to (1) results in which $\theta$ has been substituted by

$$
\theta_{\mathrm{eff}}=\theta+\beta\left(R_{S}+R_{D}\right)-\beta R_{D} \frac{V_{D S_{\mathrm{cff}}}}{V_{G S}-V_{T}} .
$$

The effects of carriers velocity saturation $\nu_{s}$ can be introduced by dividing the right-hand side of (1) by $1+V_{D S_{\text {crf }}} \mu /\left(2 \nu_{s} L\right)$ [4],[5]. Again, this can be approximated by replacing in (1) $\theta$ by

$$
\begin{aligned}
\theta_{\mathrm{eff}} & =\theta+\beta\left(R_{D}+R_{S}\right)+\left(\frac{\mu}{2 \nu_{s} L}-\beta R_{D}\right) \frac{V_{D S_{\mathrm{cff}}}}{V_{G S}-V_{T}} \\
& =\theta_{O}+\theta_{e} \frac{V_{D S_{\mathrm{cff}}}}{V_{G S}-V_{T}}
\end{aligned}
$$

where both effects-series resistances and carriers velocity saturation-are included. Parameters $\theta_{0}=\theta+\beta\left(R_{D}+R_{S}\right)$ and $\theta_{e}=\left(\mu / 2 \nu_{s} L\right)-\beta R_{D}$ have been defined for convenience. Substituting (3) into (1) yields

$$
I_{D S}=\beta \frac{V_{G S}-V_{T}\left(V_{S B}\right)-\frac{1}{2} V_{D S_{\mathrm{cff}}}}{1+\theta_{o}\left(V_{G S}-V_{T}\left(V_{S B}\right)\right)+\theta_{e} V_{D S_{\mathrm{cff}}}} .
$$


Differentiating (4) yields the following mismatch model:

$$
\begin{aligned}
\frac{\Delta I_{D S}}{I_{D S}}= & \frac{\Delta \beta}{\beta}+\frac{1}{I_{D S}} \frac{\partial I_{D S}}{\partial V_{T}}\left(\Delta V_{T 0}+\frac{\partial V_{T}}{\partial \gamma} \Delta \gamma\right) \\
& +\frac{1}{I_{D S}} \frac{\partial I_{D S}}{\partial \theta_{o}} \Delta \theta_{o}+\frac{1}{I_{D S}} \frac{\partial I_{D S}}{\partial \theta_{e}} \Delta \theta_{e}
\end{aligned}
$$

where the set of five mismatch parameters $\left\{\Delta \beta / \beta, \Delta V_{T 0}, \Delta \gamma\right.$, $\Delta \theta_{o}$, and $\left.\Delta \theta_{e}\right\}$ characterizes transistor mismatch for any bias point. Note that $\Delta \theta_{\circ}$ is the $\Delta \theta_{\text {eff }}$ mismatch in ohmic region

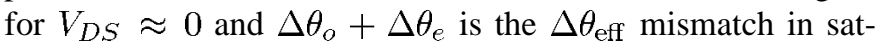
uration. In order to characterize the five mismatch parameters $\left\{\Delta \beta / \beta, \Delta V_{T 0}, \Delta \gamma, \Delta \theta_{o}, \Delta \theta_{e}\right\}$, (5) needs to be measured in ohmic region (with $V_{D S} \approx 0$ ) to extract $\Delta \theta_{o}$, and in saturation to extract $\Delta \theta_{o}+\Delta \theta_{e}$.

\section{Mismatch ChaRACTERIZATION Results}

A mismatch characterization chip including 30 different NMOS and PMOS transistor arrays of different sizes was fabricated in the ES2 1.0- $\mu \mathrm{m}$ CMOS process [6]. Transistor widths were $W=40,20,10,5,2.5$, and $1.25 \mu \mathrm{m}$ while lengths were $L=40,10,4,2$, and $1 \mu \mathrm{m}$. Each array contained 30 transistor pairs. For each pair, four $I-V$ curves were measured

$$
\begin{array}{llll}
\text { Curve 1: } & V_{D S}=0.1 \mathrm{~V} & V_{S B}=0 \mathrm{~V} & V_{G S} \in[1.5,5 \mathrm{~V}] \\
\text { Curve 2: } & V_{D S}=0.1 \mathrm{~V} & V_{G S}=3 \mathrm{~V} & V_{S B} \in[0,2 \mathrm{~V}] \\
\text { Curve 3: } & V_{D S}=4 \mathrm{~V} & V_{S B}=0 \mathrm{~V} & V_{G S} \in[1.5,5 \mathrm{~V}] \\
\text { Curve 4: } & V_{D S}=4 \mathrm{~V} & V_{G S}=3 \mathrm{~V} & V_{S B} \in[0,2 \mathrm{~V}] .
\end{array}
$$

First, the large-signal parameters $\beta, V_{T 0}, \gamma, \theta$ and $\phi$ were extracted for each curve using nonlinear curve fitting techniques [7]. These parameters were used to compute the partial derivatives in (5) for each of the four measured curves. Afterwards, four versions of (5) were obtained, one for each of the curves in (6), and were fitted simultaneously to the measured data $\Delta I_{D S} / I_{D S}$ resulting in a unique set of mismatch parameters for each transistor pair $\left\{\Delta \beta / \beta, \Delta V_{T 0}, \Delta \gamma, \Delta \theta_{o}, \Delta \theta_{e}\right\}$ valid for saturation and ohmic regions. Statistical characterization of these five parameters results in five standard deviations $\sigma_{\beta}, \sigma_{V_{T 0}}, \sigma_{\gamma}, \sigma_{\theta_{o}}, \sigma_{\theta_{e}}$ and ten correlation coefficients for each transistor size, which can be used to predict the current mismatch variance

$$
\begin{aligned}
\sigma^{2}\left(\frac{\Delta I_{D S}}{I_{D S}}\right)= & \sigma_{\beta}^{2}+\left(\frac{1}{I_{D S}} \frac{\partial I_{D S}}{\partial V_{T}}\right)^{2} \\
& \cdot\left(\sigma_{V_{T 0}}^{2}+\left(\frac{\partial V_{T}}{\partial \gamma}\right)^{2} \sigma_{\gamma}^{2}\right) \\
& +\left(\frac{1}{I_{D S}} \frac{\partial I_{D S}}{\partial \theta_{o}}\right)^{2} \sigma_{\theta_{o}}^{2}+\left(\frac{1}{I_{D S}} \frac{\partial I_{D S}}{\partial \theta_{e}}\right)^{2} \sigma_{\theta_{e}}^{2} \\
& + \text { correlation terms. }
\end{aligned}
$$

Table I shows the extracted large-signal values for $\theta_{o}$ and $\theta_{e}$ for all NMOS transistor sizes. Fig. 1 compares the measured
TABLE I

EXTRACTED VALUES FOR $\left(\theta_{o}, \theta_{e}\right)$ IN $V-1$

\begin{tabular}{llllll}
\hline$w$ & $t=40 \mu \mathrm{m}$ & $t=10 \mu \mathrm{m}$ & $t=4 \mu \mathrm{m}$ & $t=2 \mu \mathrm{m}$ & $t=1 \mu \mathrm{m}$ \\
\hline $40 \mu \mathrm{m}$ & $0.08,-0.05$ & $0.09,-0.04$ & $0.12,0.03$ & $0.17,0.24$ & $0.31,1.90$ \\
$20 \mu \mathrm{m}$ & $0.08,-0.05$ & $0.09,-0.04$ & $0.11,0.04$ & $0.16,0.23$ & $0.27,1.73$ \\
$10 \mu \mathrm{m}$ & $0.08,-0.05$ & $0.09,-0.04$ & $0.11,0.03$ & $0.15,0.22$ & $0.25,1.51$ \\
$5 \mu \mathrm{m}$ & $0.07,-0.05$ & $0.08,-0.03$ & $0.10,0.03$ & $0.14,0.20$ & $0.23,1.28$ \\
$2.5 \mu \mathrm{m}$ & $0.07,-0.04$ & $0.08,-0.03$ & $0.10,0.02$ & $0.13,0.17$ & $0.21,0.94$ \\
$1.25 \mu \mathrm{m}$ & $0.06,-0.04$ & $0.07,-0.03$ & $0.09,0.02$ & $0.12,0.14$ & $0.21,0.77$ \\
\hline
\end{tabular}
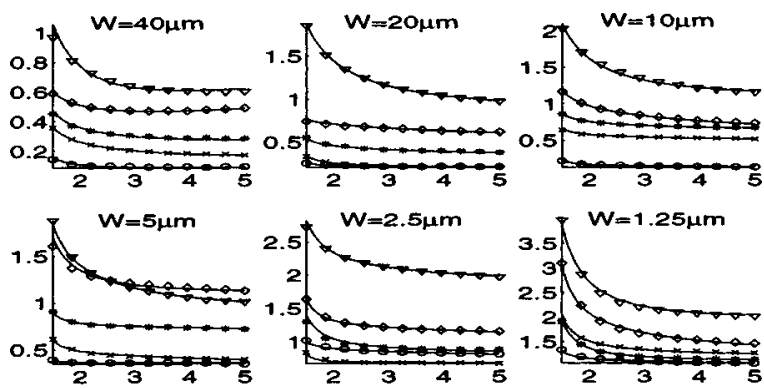

(a)
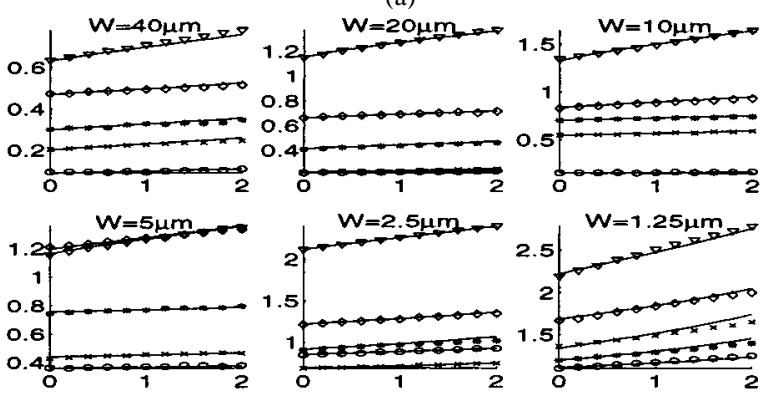

(b)
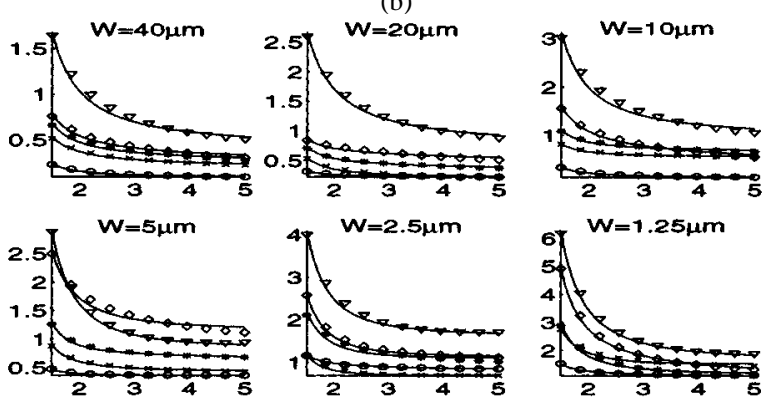

(c)
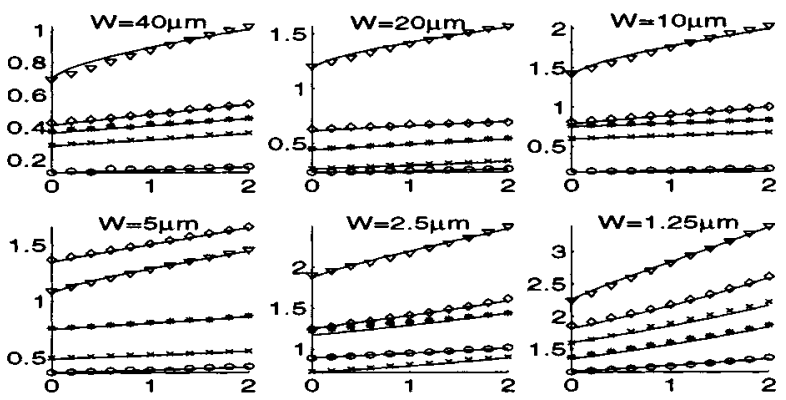

(d)

Fig. 1. Measured versus predicted current mismatch $\sigma\left(\Delta I_{D S} / I_{D S}\right)$ (in \%) for all NMOS transistor sizes for (a) Curve 1, (b) Curve 2, (c) Curve 3, and (d) Curve 4. Symbols denote different transistor lengths: circles $L=40 \mu \mathrm{m}$, crosses $L=10 \mu \mathrm{m}$, stars $L=4 \mu \mathrm{m}$, diamonds $L=2 \mu \mathrm{m}$, and triangles $L=1 \mu \mathrm{m}$. 

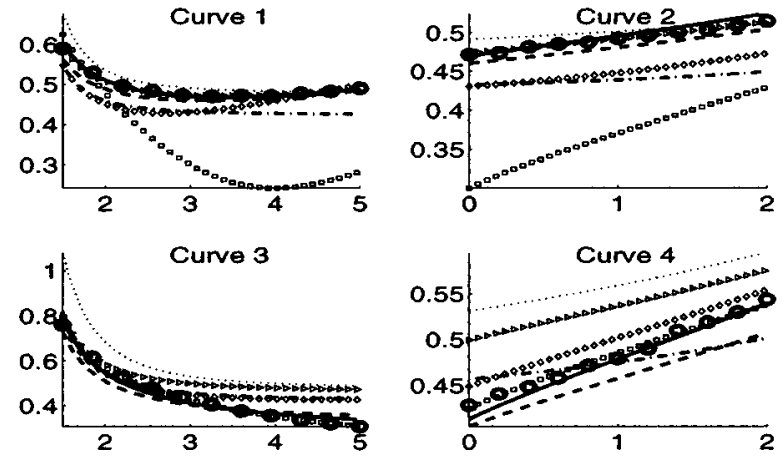

Fig. 2. Comparison of $\sigma\left(\Delta I_{D S} / I_{D S}\right)$ for all four measured curves using different mismatch models, for NMOS transistors of size $W=40 \mu \mathrm{m}, L=2$ $\mu \mathrm{m}$.

values of $\sigma\left(\Delta I_{D S} / I_{D S}\right)$ (shown with symbols) with those predicted by (7) for the four measured curves of (6), for all 30 NMOS transistor sizes. As can be seen, the agreement between measured and predicted mismatch is excellent. Such agreement cannot be achieved using conventional mismatch models for such a wide range of transistor sizes, including short-channel $(1 \mu \mathrm{m})$ transistors.

In order to compare with other models, Fig. 2 shows $\sigma\left(\Delta I_{D S} / I_{D S}\right)$ for NMOS transistors of size $W=40 \mu \mathrm{m}$, $L=2 \mu \mathrm{m}$, for all four measured curves. Circles are measured values. Continuous lines are predicted values using the model proposed in this letter, including all correlation coefficients. Dashed lines are predicted with the same model, but considering only the three most relevant correlation coefficients $\left(r_{\left(\Delta \beta, \Delta \theta_{o}\right)}, r_{\left(\Delta \beta, \Delta \theta_{e}\right)}, r_{(\Delta \beta, \Delta \gamma)}\right)$. Dotted lines correspond to extracting only $\left\{\Delta \beta / \beta, \Delta V_{T 0}, \Delta \gamma\right\}$ for ohmic region, and use the resulting $\left\{\sigma_{\beta}, \sigma_{V_{T 0}}, \sigma_{\gamma}\right\}$ (without correlation coefficients) to predict $\sigma\left(\Delta I_{D S} / I_{D S}\right)$ in both ohmic and saturation regions. This is the method used by Pelgrom [1]. Dashed-dotted lines correspond to extracting $\left\{\Delta \beta / \beta, \Delta V_{T 0}, \Delta \gamma\right\}$ for both regions, and use the resulting $\left\{\sigma_{\beta}, \sigma_{V_{T 0}}, \sigma_{\gamma}\right\}$ (without correlation coefficients) to predict $\sigma\left(\Delta I_{D S} / I_{D S}\right)$ in both regions. Lines with little squares correspond to extracting $\left\{\Delta \beta / \beta, \Delta V_{T 0}, \Delta \gamma, \Delta \theta\right\}$ in saturation, and use the resulting $\left\{\sigma_{\beta}, \sigma_{V_{T 0}}, \sigma_{\gamma}, \sigma_{\theta}\right\}$ (and correlation coefficients) to predict $\sigma\left(\Delta I_{D S} / I_{D S}\right)$ in both regions [3]. Lines with little triangles are the same but extracting $\left\{\Delta \beta / \beta, \Delta V_{T 0}, \Delta \gamma, \Delta \theta\right\}$ in ohmic region. And finally, lines with diamonds are the same but extracting $\left\{\Delta \beta / \beta, \Delta V_{T 0}, \Delta \gamma, \Delta \theta\right\}$ for both regions.

\section{REFERENCES}

[1] M. J. M. Pelgrom, A. C. J. Duinmaijer, and A. P. G. Welbers, "Matching properties of MOS transistors," IEEE J. Solid State Circuits, vol. 24, pp. 1433-1440, 1989.

[2] P. E. Allen and D. R. Holberg, CMOS Analog Design, New York: Holt, Rinehart and Winston Inc., 1987.

[3] J. Bastos, M. Steyaert, A. Pergoot, and W. Sansen, "Mismatch characterization of submicron MOS transistors," Analog Integrated Circuits and Signal Processing, vol. 12, pp. 95-106, 1997.

[4] Y. Tsividis, Operation and Modeling of the MOS Transistor, New York: McGraw-Hill, 1988.

[5] U. Cilingiroglu, Systematic Analysis of Bipolar and MOS Transistors. Norwood, MA: Artech House, 1993.

[6] T. Serrano-Gotarredona and B. Linares-Barranco, "Systematic width-and-length dependent cmos transistor mismatch characterization and Simulation," J. Analog Integr. Circuits Signal Process., vol. 21, no. 3, 1999.

[7] W. H. Press, B. P. Flannery, S. A. Teukolsky, and W. T. Vetterling, Numerical Recipes in C. The Art of Computing. Cambridge, U.K.: Cambridge Univ. Press, 1988. 